\title{
Mechanistic evidence for a front side, SNi-type reaction in a retaining glycosyltransferase
}

Seung Seo Lee, Sung You Hong, James C. Errey, Atsushi Izumi, Gideon J. Davies and Benjamin G. Davis

School of Chemistry, University of Southampton, Southampton UK SO17 1BJ. Email: S.S.Lee@soton.ac.uk

Please cite this paper as:

Nature Chemical Biology, 2011, (7), 631-638

The publisher's version of this paper is available here:

http://dx.doi.org/10.1038/nchembio.628

\section{Related articles by Dr Seung Seo Lee can be found below:}

Seung Seo Lee, Sung You Hong, James C. Errey, Atsushi Izumi, Gideon J. Davies, and Benjamin G. Davis (2011) Mechanistic evidence for a front-side, $\mathbf{S}_{\underline{N}} \underline{\mathrm{i}}$-type reaction in a retaining glycosyltransferase, Nature Chemical Biology, 7, 631-638 - highlighted in ChemBioChem (2011) 12, 2540-2542 (DOI:10.1038/NCHEMBIO.628)

James C. Errey, Seung Seo Lee, Robert P. Gibson, Carlos Martinez Fleites, Conor S. Barry, Pierre M. J. Jung, Anthony O'Sullivan, Benjamin G. Davis and Gideon J. Davies (2010) Mechanistic Insight into Enzymatic Glycosyltransfer with Retention of Configuration through Analysis of Glycomimetic Inhibitors, Angewandte Chemie International Edition in English, 49, 1234 - 1237 (DOI:10.1002/anie.200905096). 


\section{Mechanistic evidence for a front side, $\mathrm{S}_{\mathrm{N}} \mathrm{i}$-type reaction in a retaining glycosyltransferase}

Seung Seo Lee ${ }^{1}$, Sung You Hong ${ }^{1,3}$, James C. Errey ${ }^{1}$, Atsushi Izumi ${ }^{2}$, Gideon J. Davies ${ }^{2}$ and Benjamin G. Davis ${ }^{1 *}$

${ }^{1}$ Department of Chemistry, University of Oxford, Chemistry Research Laboratory, Mansfield Road Oxford OX1 3TA, UK

${ }^{2}$ Department of Chemistry, The University of York, Heslington, York, YO10 5DD, UK

* e-mail: ben.davis@chem.ox.ac.uk

${ }^{3}$ Present address: School of Nano-Bioscience and Chemical Engineering (NBC), Ulsan National Institute of Science and Technology (UNIST), Ulsan 689-798, South Korea 


\begin{abstract}
144 words
A previously determined crystal structure of the ternary complex of trehalose-6phosphate synthase identified a putative transition state-like arrangement based upon validoxylamine-A-6-O-phosphate and uridine diphosphate in the active site. Here, linear free energy relationships confirm that these inhibitors are synergistic transition state mimics, supporting frontal face nucleophilic attack involving hydrogen bonding between leaving group and nucleophile. Kinetic isotope effects indicate a highly dissociative oxocarbenium ion-like transition state. Leaving group ${ }^{18} \mathrm{O}$ effects identified both isotopically-sensitive bond cleavage and support the existence of a hydrogen bond between the nucleophile and departing group. Brønsted analysis of nucleophiles and Taft analysis highlights participation of the nucleophile in the transition state, also consistent with a front face mechanism. Together, these comprehensive, quantitative data substantiate this unusual reaction mechanism in an enzyme. Its discovery in nature should prompt useful reassessment of many biocatalysts and their substrates and inhibitors.
\end{abstract}




\section{INTRODUCTION}

Glycosyltransferases (GTs) are among the most important enzymes in the field of glycobiology. They are responsible for the biosynthesis of glycans and glycoconjugates, which play a vital role in signaling, recognition, pathogenesis and bacterial cell wall formation. ${ }^{1}$ However, the catalytic mechanism of GTs that utilize nucleoside diphosphate sugars (UDP-glucose, GDP-mannose, etc) remains mostly unclear. ${ }^{1}$ The reactions of the GTs that result in the retention of stereochemistry at the anomeric center (retaining GTs) are especially poorly understood, whilst inverting GTs are suggested to utilize a single displacement mechanism with an oxocarbenium ion-like transition state and an asynchronous $\mathrm{S}_{\mathrm{N}} 2$ mechanism; a mechanism which is supported by several mechanistic studies and kinetic isotope effects measurements on inverting GTs $s^{2,3}$ as well as by numerous $3-\mathrm{D}$ structures ${ }^{1}$. In contrast, the kinetic and structural studies of retaining GTs have been unsuccessful in providing unambiguous evidence for their mode of action ${ }^{1}$. With analogy to the double displacement mechanism of retaining glycoside hydrolases $(\mathrm{GHs})^{4}$ that involves the formation of a covalent intermediate with configurational inversion, followed by the hydrolysis of the intermediate with another inversion, the retaining GTs are suggested to follow a double displacement mechanism. However, many data argue at odds with the double displacement pathway. For instance, although some elegant experiments using mutant enzymes are able to highlight intermediates $^{5,6}$, for example on distant nucleophiles, ${ }^{5}$ or perform chemical rescue ${ }^{7}$, attempts to trap the covalent glycosyl-enzyme intermediate and other types of relevant intermediates in wild-type enzymes are unsuccessful. ${ }^{5,8}$ Moreover a long list ${ }^{9-15}$ (see also Supplementary Results) of structural data of retaining glycosyltransferases exists, that suggests few amino acid side chains suitably positioned to act as a nucleophile in 
the active site in such a mechanism. Amino acid sequence alignments fail to show any consistently conserved amino acid residues for such an important role. In GHs, where such a mechanism is well documented, the nucleophilic and acid/base residues within the enzyme family are essentially invariant. ${ }^{1}$

As an alternative possibility, the "internal return-like" mechanism, so called $\mathrm{S}_{\mathrm{N}} \mathrm{i}$ like mechanism has been suggested, depicting the nucleophilic attack from the same face as the leaving group departure. ${ }^{1,10,15}$ The concept of a general internal nucleophilic

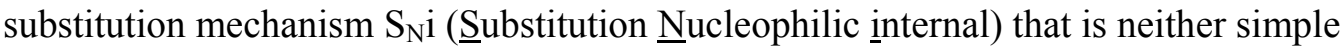
$\mathrm{S}_{\mathrm{N}} 1$ nor $\mathrm{S}_{\mathrm{N}} 2$ was first invoked to explain unusual stereochemical outcomes of simple alkyl halides; ${ }^{16}$ this so-called Ingold-type nomenclature focuses on defining transformation type. This mechanism is classically invoked in the characterization of the reactive stereochemical course of secondary alkyl chlorosulfites which leads to retention when the nucleophile and leaving group are constrained on the same face; importantly these early studies highlighted that when constraint was removed then attack occurred from the other face. This mechanism is also observed in carbohydrate chemistry. Of direct relevance, such a mechanism for glycosyltransfer was first proposed, in the absence of any enzyme, to explain the retention of anomeric stereochemistry in the solvolysis of $\alpha$-glucosyl fluoride by mixtures of ethanol and trifluorethanol. ${ }^{17}$ Theoretical studies suggest that such an internal return mechanism can provide an energetically plausible pathway inside enzymes as a result of active site geometrical constraints $^{18}$. An internal return-like or $\mathrm{S}_{\mathrm{N}} \mathrm{i}$-like mechanism is also suggested based on the ternary complex structure of a retaining galactosyltransferase from Neisseria meningitidis containing acceptor and donor substrate analogues and kinetic study of mutants ${ }^{10}$. A series of reports on an archetypal, non-Leloir glycosyltransferase, trehalose phosphorylase, have postulated that this unusual mechanism might be operating based 
on kinetic data. ${ }^{19,20}$ In a recent study, a change in kinetic and chemical mechanism is also suggested, from a double displacement to a sequential "front side" mechanism when a retaining glycosidase, sucrose phosphorylase, is engineered to possess synthetic activity. ${ }^{21}$ Nonetheless, no study has yet gathered comprehensive mechanistic evidence.

The $\mathrm{S}_{\mathrm{N}} \mathrm{i}$-like mechanism involves a frontal-face nucleophilic attack, thereby leading to net retention in the nucleophilic substitution, which features an "open" transition state (TS) ${ }^{17}$. This 'open' TS is expected to be highly dissociative, possessing a considerable oxocarbenium ion-character ${ }^{18}$. An interaction, possibly hydrogen bonding, between the leaving group and the incoming nucleophile is predicted, which should lead the nucleophile to the same face as the leaving group while assisting leaving group dissociation $^{1,17}$. Recently, we described the X-ray structure ${ }^{22}$ of a ternary complex (Figure 1) of a retaining glycosyltransferase, trehalose-6-phosphate synthase (OtsA) containing uridine diphosphate (UDP) and a bisubstrate analogue, validoxylamineA-6'O-phosphate (VA6P (1), Figure 1d). OtsA transfers a glucosyl moiety from a donor substrate uridine diphosphate glucose (UDP-Glc) to the 1-hydroxyl group of the acceptor substrate glucose-6-phosphate (Glc6P) to form an $\alpha, \alpha-1,1$-linkage, yielding the product $\alpha, \alpha-1,1$-trehalose-6-phosphate (Figure 1a). OtsA belongs to a sequence based family GT20 and possesses a GT-B fold ${ }^{23}$. Kinetically, OtsA utilizes a sequential ordered bi-bi mechanism, in which the donor, UDP-Glc binds to the active site first, followed by the binding of the acceptor Glc6P, prior to reaction. VA6P displays competitive inhibition with regard to the donor UDP-Glc and its potency increases considerably in the presence of UDP. This synergistic inhibition is believed to show the plausible geometry and interplay of a leaving group and a nucleophile that can happen in the transition state (Figure 1b). Moreover, the 3-D structure of this complex shows that a hydrogen bond exists between the leaving group oxygen of UDP and the 
nucleophile mimic of the sugar moiety (Figure 1c). This and other structures ${ }^{15}$ are consistent with the suggestions that the $\mathrm{S}_{\mathrm{N}} \mathrm{i}$-like mechanism requires a hydrogen bonding that can direct the nucleophile to the same face as the leaving group ${ }^{17}$. Computational studies also suggest that hydrogen bonding between the leaving group and the nucleophile contributes to stabilizing the transition state of an $\mathrm{S}_{\mathrm{N}} \mathrm{i}$ mechanism ${ }^{18}$. Also, the flattened geometry of the leaving group pyranose ring mimic in this structure (VA6P) is consistent with oxocarbenium ion character. Overall, although our previous ternary complex structure is suggestive of an $\mathrm{S}_{\mathrm{N}} \mathrm{i}$ mechanism, more experimental data to test the mechanistic hypotheses are required.

In this report, we present what we believe is compelling evidence that observations in our ternary complex reflect true aspects of the transition state (TS). We have probed the characteristics of the putative TS structure and the participation of the nucleophile in the TS by means of kinetic isotope effects (KIE) and linear free energy relationships (LFER) on a range of substrates; together these data implicate both the leaving group of the donor and the acceptor nucleophile during the TS and suggest a front side, $\mathrm{S}_{\mathrm{N}} \mathrm{i}$-type mechanism.

\section{RESULTS}

\section{Confirming transition state mimicry}

LFER can be used to probe the sensitivity of reaction rates to electronic factors, such as the electronegativity or acidity of atoms near the reaction center ${ }^{24}$ and can provide a rigorous test of the transition state mimicry of inhibitors. ${ }^{25}$ Using the latter strategy, we compared the effects of identical structural change on the stabilization of the transition state of the reaction (reflected in $\mathrm{k}_{\mathrm{cat}} / \mathrm{K}_{\mathrm{m}}$ ) and on the binding affinity of potential TS 
mimics (reflected in $\mathrm{K}_{\mathrm{i}}$ ). This can be achieved either by systematically changing the structure of the substrates (giving $\mathrm{k}_{\mathrm{cat}} / \mathrm{K}_{\mathrm{m}}$ values) and analogously of the inhibitors (giving $\mathrm{K}_{\mathrm{i}}$ values) or by mutating amino acid residues in the active site of enzymes, both parameters measured with the same substrate and inhibitor. ${ }^{25}$ Thus, we generated active site mutants of OtsA and measured all relevant kinetic parameters. Initial KIEs observed through $\mathrm{k}_{\mathrm{cat}} / \mathrm{K}_{\mathrm{m}}$ (see below) confirmed that the chemical step was sufficiently isotopically sensitive in the mechanism of OtsA, showing that $\mathrm{k}_{\text {cat }} / \mathrm{K}_{\mathrm{m}}$ reflected the TS of the chemical reactions, and highlighted this as a highly suitable enzymatic system. To test whether the inhibition by validoxylamine-6-phosphate (VA6P) in the presence of UDP was relevant to the TS of the reaction, we expressed wild-type and active site mutant variants of trehalose-6-phosphate synthase (OtsA) in E. coli and purified them by Ni-affinity chromatography. Kinetic parameters of a donor substrate, UDP-Glc, and an acceptor substrate, Glc6P, were measured by the coupled spectrophotometric assay method $^{26}$ (Supplementary Table 1).

Attempts were made to measure the inhibition constants of VA6P for mutants without UDP. At $5 \mathrm{mM}$ concentration of VA6P, inhibition was less than $10 \%$ with the D130A mutant and other mutants showed no significant inhibition. Thus, except for the WT enzyme, 5 mM VA6P alone did not significantly inhibit any mutants giving determined $\mathrm{K}_{\mathrm{i}}$ values that in all cases were at least two orders of magnitude higher (Supplementary Table 1). Nonetheless, more potent inhibition by VA6P could be measured in the presence of UDP, confirming the synergistic inhibition of VA6P and UDP. Firstly, radioactivity assay employing ${ }^{3} \mathrm{H}$-labelled UDP-Glc measured inhibition constants of UDP with respect to the donor substrate, UDP-Glc, for the wild type enzyme and active site variants (See Methods). Next, radioactivity assay measured $\mathrm{K}_{\mathrm{i}}$ values of VA6P in the presence of UDP. $\mathrm{K}_{\mathrm{i}}$ values could be measured with the mutants 
and ranged from $2.7 \mu \mathrm{M}$ for the wildtype to $7.6 \mathrm{mM}$ for the least active enzyme mutant R262K (Supplementary Table 1 and Supplementary Figure 2). Apparent $\mathrm{K}_{\mathrm{i}}$ values of VA6P were measured in the presence of UDP at a concentration corresponding to twice the $\mathrm{K}_{\mathrm{i}}$ values of UDP for each mutant. At this concentration of UDP, the occupancy of UDP within the active site was substantial and yet the residual activity was measurable by the sensitive radioactivity assay. Logarithms of $\mathrm{K}_{\mathrm{i}}$ values plotted against those of $\mathrm{K}_{\mathrm{m}} / \mathrm{k}_{\text {cat }}$ values, revealed a significant relationship with a correlation coefficient $\left(\mathrm{r}^{2}\right)$ of 0.94 and a slope of 0.98 (Figure 2a). Generally, strong correlation with a slope close to unity is considered to indicate the meaningful mimicry of transition state ${ }^{25}$ and therefore this LFER strongly suggested that synergistic inhibitors, VA6P and UDP, formed an in situ transition state analogue at the active site. VA6P alone gave only a very poor correlation (Figure $2 \mathrm{~b}$ ) and only at high concentrations ( $>5 \mathrm{mM})$; UDP alone (Figure $2 \mathrm{c}$ ) similarly gave no clear correlation. In situ formation of a TS analogue is not rare and is observed in studies of $\beta$-phosphoglucomutase ${ }^{27}$ and a retaining GT, trehalose phosphorylase $^{19}$. Consequently, it is likely that the structure of the OtsA ternary complex $^{22}$ reflects characteristics of the true transition state.

\section{Kinetic analysis of the donor substrate}

A powerful method to probe the oxocarbenium ion character in the reaction coordinate is through kinetic isotope effects (KIEs). ${ }^{28}$. Since isotopic change is very sensitive to vibrational mode and frequency, the resulting subtle variation in reaction rate upon isotopic substitution provides insight into the change in bond order and geometry around the atom subjected to isotopic substitution while the reaction is going from ground state to the TS. If the transition state has a looser structure or weaker bonding than the ground state, then heavy isotope substitution will result in a slower rate, giving 
a normal KIE $\left(\mathrm{k}_{\text {light }} / \mathrm{k}_{\text {heavy }}>1\right)$. In contrast, a TS with a more constricted environment or stronger bonding between atoms results in a faster rate with heavy isotope substitution, giving an inverse KIE $\left(\mathrm{k}_{\text {light }} / \mathrm{k}_{\text {heavy }}<1\right)$. Thus, both the mode and magnitude of KIEs can provide detailed information about TS structure. It is important to note that the reaction coordinate and aspects of reversibility and commitment ${ }^{29}$ (see also below) can also modulate KIE.

We determined multiple KIEs under competitive conditions using the isotopically labeled donor substrates shown in Table 1 . These methods employed radioisotopes as heavy atoms and remote labels ${ }^{30,31}$, and yielded KIEs based upon $\mathrm{k}_{\mathrm{cat}} / \mathrm{K}_{\mathrm{m}}{ }^{32}$, which reflected the first irreversible step. Thus, the donor substrate, UDP-Glc, was isotopically labeled at a range of sites with $\left[1^{\prime \prime}-{ }^{3} \mathrm{H}\right],\left[1^{\prime \prime}-{ }^{2} \mathrm{H}, 5-{ }^{3} \mathrm{H}\right],\left[2^{\prime \prime}-{ }^{2} \mathrm{H}, 5-{ }^{3} \mathrm{H}\right]$, $\left[1^{\prime \prime}-{ }_{-}^{14} \mathrm{C}\right],\left[1^{\prime \prime}-{ }^{18} \mathrm{O}, 5-{ }^{3} \mathrm{H}\right],\left[6^{\prime \prime}-{ }_{-}^{3} \mathrm{H}\right],\left[6^{\prime \prime}{ }_{-}{ }^{14} \mathrm{C}\right]$, and $\left[5-{ }^{3} \mathrm{H}\right]$ (Table 1). These were synthesized enzymatically or chemoenzymatically from appropriately labeled glucose (see Supplementary Methods). Since the 6" position is three bonds away from the reaction center, the $\mathrm{KIE}$ from ${ }^{14} \mathrm{C}$ at this position was assumed to be negligible ${ }^{33}$ and was therefore used as a reference. Thus, KIEs of $\left[1^{\prime \prime}-{ }^{3} \mathrm{H}\right],\left[1^{\prime \prime}-{ }^{2} \mathrm{H}, 5-{ }^{3} \mathrm{H}\right],\left[2^{\prime \prime}-{ }^{2} \mathrm{H}, 5-{ }^{3} \mathrm{H}\right]$, $\left[1^{\prime \prime}-{ }_{-}^{18} \mathrm{O}, 5-{ }^{3} \mathrm{H}\right],\left[6^{\prime \prime}-{ }_{-}^{3} \mathrm{H}\right]$, and $\left[5-{ }^{3} \mathrm{H}\right]$ measured in pair with $\left[6^{\prime \prime}-{ }_{-}^{14} \mathrm{C}\right]$ as a competitive light isotopic substrate provided a precise internal standard. Similarly, the negligible effect of a $\left[5-{ }^{3} \mathrm{H}\right]$ label was confirmed and so justified its use as a concomitant silent remote label in $\left[1^{\prime \prime}-{ }_{-}^{2} \mathrm{H}, 5-{ }^{3} \mathrm{H}\right],\left[2^{\prime \prime}-{ }^{2} \mathrm{H}, 5-{ }^{3} \mathrm{H}\right]$ and $\left[1^{\prime \prime}-{ }^{18} \mathrm{O}, 5-{ }^{3} \mathrm{H}\right]$ KIEs. A $\left[1^{\prime \prime}{ }_{-}{ }^{14} \mathrm{C}\right]$ KIE was measured with $\left[6^{\prime \prime}-{ }^{3} \mathrm{H}\right]$ labeled substrate as a competitive light isotope label and corrected for remote effects by the latter.

In the retaining GT reaction, the first irreversible step was expected to reflect the departure of a leaving group and the nucleophilic participation of the acceptor nucleophile as suggested by the LFER through TS analogy. For KIE to be a meaningful 
tool revealing chemical transformation, it is necessary for the chemical step to be the first irreversible step so that the measured KIE is fully intrinsic. Otherwise, measured KIEs will be significantly lower than intrinsic values, misleading the interpretation. Therefore in many cases it has been necessary to measure commitment factors of the reaction. Commitment factors measure the ratio of the rate of the bond-forming (or breaking) step to those of all non-chemical steps, thus revealing how contributory the chemical step is. A correction of experimental KIEs based on measured commitment factors can be made to obtain intrinsic $\mathrm{KIEs}^{32}$. In general, there are two types of commitment factors, forward and reverse. The OtsA catalyzed reaction is virtually irreversible under the assay conditions and thus reverse commitment can be considered zero.

D-Glucose-6-phosphate (Glc6P) was used as an acceptor substrate only for the measurement of a $\left[1^{\prime \prime}-{ }^{3} \mathrm{H}\right] \mathrm{KIE}$. Although the $\left[1^{\prime \prime}-{ }_{-}^{3} \mathrm{H}\right] \mathrm{KIE}$ measured with the natural acceptor Glc6P was significantly large (1.205 \pm 0.002 s.d.), we also chose the slower and less efficient acceptor substrate, 2-deoxy-D-glucose-6-phosphate (2dGlc6P, (2)) (two-fold less $\mathrm{k}_{\text {cat }}$ and 8 fold higher $\mathrm{K}_{\mathrm{m}}$ ) to complete our set of multiple KIEs and to make the chemical step more dominant (Table 1). Measurement of a full set of KIEs $\left(\left[1^{\prime \prime}-{ }^{3} \mathrm{H}\right]\right.$ and $\left.\left[1^{\prime \prime}-{ }_{-}^{2} \mathrm{H}\right]\right)$ with the less active acceptor substrate $2 \mathrm{dGlc} 6 \mathrm{P}(2)$ ensured the determination of intrinsic KIEs; indeed, the $\left[1^{\prime \prime}-{ }^{3} \mathrm{H}\right] \mathrm{KIE}$ of 1.284 obtained using $2 \mathrm{dG} 6 \mathrm{P}$ (2) was larger than that measured with Glc6P and among the largest reported. Moreover, high $\mathrm{K}_{\mathrm{m}}$ values (53 mM, in this case) implicated weak binding and very little commitment $^{33}$. Together these data indicated that the KIEs measured with 2dGlc6P (2) can be considered intrinsic.

The $\alpha$-secondary $\left[1^{\prime \prime}-{ }^{3} \mathrm{H}\right]$ KIE was measured with a magnitude of 1.284 . Such large $\alpha$-secondary ${ }^{3} \mathrm{H}$ ( or ${ }^{2} \mathrm{H}$ ) KIEs are an indication of oxocarbenium ion character at 
the transition state due to the change of hybridization of anomeric carbon from $\mathrm{sp}^{3}$ to $\mathrm{sp}^{2}$, which creates a less restricted environment around the C-H bond enabling out-ofplane bending freedom of the H(D) atom. ${ }^{34}$ The large $\beta$-secondary KIE from $\left[2^{\prime \prime}-{ }^{2} \mathrm{H}\right]$ of 1.164 further supported this change. The $\beta$-secondary deuterium KIE measured the hyperconjugation between the $2^{\prime \prime}$-hydron (any of ${ }^{1} \mathrm{H},{ }^{2} \mathrm{H}$ or ${ }^{3} \mathrm{H}$ ) and the electron deficient $\mathrm{C}^{\prime \prime}$ of the oxocarbenium, which stabilizes the $\mathrm{TS}^{35}$. This hyperconjugation can be described as electron donation from the sigma bond between $\mathrm{H}_{2}^{\prime \prime}$ and $\mathrm{C}^{\prime \prime}$ to an empty p-orbital of $\mathrm{C}^{\prime \prime}$ at the $\mathrm{TS}$, which results in a weakened $\mathrm{C} 2^{\prime \prime}-\mathrm{H} 2^{\prime \prime}$ bond, leading to a normal (>1) KIE. Such electron donation is highly dependent on the torsion angle between $\mathrm{C}^{\prime \prime}-\mathrm{H} 2^{\prime \prime}$ and the p-orbital of $\mathrm{C}^{\prime \prime}$. If the pyranose ring is flattened on going to the TS, hyperconjugation is more efficient, leading to a large KIE. Together large normal $\left[1^{\prime \prime}-{ }^{3} \mathrm{H}\right] \mathrm{KIE}$ and $\left[2^{\prime \prime}-{ }^{2} \mathrm{H}\right] \mathrm{KIEs}(\alpha$ - and $\beta$-secondary, respectively) strongly indicated that the TS was considerably dissociative with a substantial oxocarbenium ion character and that the pyranose ring was flattened through $\mathrm{C}^{\prime \prime}-\mathrm{O} 5^{\prime \prime}-\mathrm{C} 1^{\prime \prime}-\mathrm{C} 2$ " (Figure 3). The $\left[1^{\prime \prime}-{ }^{14} \mathrm{C}\right] \mathrm{KIE}$ also supported these observations. $\left[1^{\prime \prime}-{ }_{-}^{14} \mathrm{C}\right] \mathrm{KIE}$ is a primary effect, normally of magnitude $1.0-1.02$ for the TS of formation of a discrete cationic intermediate $\left(D_{N} * A_{N}{ }^{36}\right), 1.025-1.060$ for a dissociative concerted mechanism $\left(A_{N} D_{N}\right)$, and $1.06-1.16$ for an associative or synchronous concerted mechanism (also, $\left.A_{N} D_{N}\right)^{28,37,38}$. Interestingly, our experimental KIE of 1.023 fell between the value ranges, and suggested an intermediate $D_{N} * A_{N}$ and dissociative $A_{N} D_{N}$ character (Figure 3 ). This result was consistent with a highly dissociative TS, but it did not distinguish between the stepwise $\left(D_{N} * A_{N}\right)$ and concerted $\left(A_{N} D_{N}\right)$ pathways. Thus although, with these experimental ${ }^{14} \mathrm{C}$ KIE data, it could be confidently stated that the TS did not have significant bond orders to either the leaving group or the incoming nucleophile, the discrete formation of an oxocarbenium ion intermediate was unclear. 
More interesting is the leaving group oxygen KIE. The $\left[1^{\prime \prime}{ }_{-}{ }^{18} \mathrm{O}\right] \mathrm{KIE}$ was determined to be 1.024 . This KIE is also primary and, in general, directly proportional to the extent of bond cleavage. ${ }^{39} \mathrm{~A}$ fully broken $\mathrm{C}-\mathrm{O}$ bond generating an anionic leaving group in glycoside hydrolysis results in a leaving group ${ }^{18} \mathrm{O}$ KIE of $1.047 .{ }^{39}$ As a comparison, leaving group ${ }^{18} \mathrm{O}$ KIEs for acid catalyzed $\mathrm{C}-\mathrm{O}$ bond cleavage presumed to proceed via fully protonated transition states are reported to be 1.023 to $1.026 .^{40,41}$ These latter measurements suggested that our measured KIE of 1.024 might be consistent with those corresponding to a protonated (at least in part) leaving group oxygen. Importantly, it should be noted that such prior values are determined using unnatural alkyloxy or aryloxy leaving groups in contrast to the phosphate leaving group, UDP, used in our case. With a phosphate leaving group, negative charge delocalization to the non-bridging oxygens or protonation instead on these non-bridging oxygens may also contribute to the observed lowering of the ${ }^{18} \mathrm{O}$ leaving group KIE. ${ }^{42}$ It may be that protonation and charge delocalization competed for the electron density liberated by the bond cleavage and the KIE of 1.024 determined here reflected both contributions, protonation and charge delocalization. Partial protonation (which was estimated to be less than $50 \%$ from the observation of Bronsted and Taft relationships, see below) appeared also to be consistent with the structure of the ternary complex and the model that hydrogen bonding between leaving group and nucleophile guides the latter to the same face as the former ${ }^{22}$. Together, these multiple KIEs were consistent with the observations in the structure of the OtsA ternary complex, its TS analogy and an $\mathrm{S}_{\mathrm{N}} \mathrm{i}$ like mechanism (Figure 4).

\section{Kinetic analysis of the acceptor substrate}


These results coupled with TS analogy suggested the involvement of both the donor and acceptor substrates, therefore the participation of acceptor substrates was also probed by LFER studies. We synthesized a panel of modified acceptor substrates, 2-deoxy (2), 3deoxy- (3), 4-deoxy- (4), 2-deoxy-2-fluoro- (7), 3-deoxy-3-fluoro- (8), 4-deoxy-4fluoro- (9), and 2-deoxy-2,2-di-fluoro-D-glucose-6-phosphate (6) using hexokinase ${ }^{43}$ along with the C-2 epimer, 2-deoxy-2-fluoro-D-mannose-6-phosphate (2FMan6P). Pseudo-single substrate steady-state kinetic experiments tested all 8 acceptor substrates (Figure 4a) in the presence of a saturating concentration of UDP-Glc and the coupled assay determined kinetic parameters (Supplementary Table 2). All modified substrates showed significant activity. The lowest $\mathrm{k}_{\text {cat }}(2,2$-diFGlc6P (6)) was only 50 -fold less than that of the natural acceptor and the corresponding $\mathrm{k}_{\mathrm{cat}} / \mathrm{K}_{\mathrm{m}} 200$-fold lower.

The x-ray crystal structure of OtsA complexed with UDP and Glc6P shows that every hydroxyl group of the acceptor makes hydrogen bonds with the enzyme active site amino acid residues directly or via water molecules. ${ }^{9}$ Monodeoxy or monodeoxyfluoro acceptors can be considered to be missing one potential hydrogen bond donor group; this implied that if used as acceptors then the penalty given to modified substrates with regard to the loss of binding affinity may be similar. Indeed, $\mathrm{K}_{\mathrm{m}}$ values, a rough estimation of enzymatic binding affinity, varied less than 2.5 -fold between all our modified substrates, representing just $0.5 \mathrm{kcal} / \mathrm{mol}$ of apparent binding energy difference. A pertinent example is $2 \mathrm{FMan} 6 \mathrm{P}$ for which the $\mathrm{k}_{\text {cat }}$ was only slightly higher than that of 2FGlc6P (7) while $\mathrm{K}_{\mathrm{m}}$ was in general agreement (within the error range), indicating that the effect of losing one hydrogen bond was approximately identical and that the orientation (equatorial or axial) of fluorine substitution had only a minor effect. The natural substrate Glc6P was excluded in all analysis since it has the potential for 
extra hydrogen bonding, resulting in extra binding energy, which cannot be in line with the desired structure-activity relationships (Supplementary Figure 2).

Firstly, we performed Brønsted-like analysis. When calculated $\mathrm{pK}_{\mathrm{a}}$ values (Supplementary Table 2 and Supplementary Methods) of the nucleophiles were plotted against $\log \left(\mathrm{k}_{\mathrm{cat}} / \mathrm{K}_{\mathrm{m}}\right)$, it revealed a strong relationship $\left(\mathrm{r}^{2}=0.98\right)$ and revealed a moderate sensitivity of the reaction to nucleophile/acceptor acidity (slope, $\beta_{\text {nuc }}=0.54 \pm 0.5$ )

(Figure 4b). Secondly, we conducted Taft-like analysis using acceptors modified only at the $\mathrm{C} 2$ position. Again, modification of the substrate at only one position was expected to give more accurate and relevant structure-activity relationships (Figure 5b). In this analysis, the natural acceptor substrate, Glc6P was again excluded for the same reasons as in the Brønsted analysis. It should be noted that polar substituent constants, $\sigma^{*} \mathrm{~s}$ for substituents at C-2 of 2dGlc6P (2), 2FGlc6P (7), 2FMan6P (5) and 2,2diFGlc6P (6) are not known and determined $\sigma^{*}$ s for $\mathrm{CH}_{3}, \mathrm{CH}_{2} \mathrm{~F}$, and $\mathrm{CHF}_{2}$ were used as the closest approximations. ${ }^{44}$ Taft-like analysis gave an excellent correlative relationship $\left(\mathrm{r}^{2}=1.0\right)$ and modest sensitivity (slope, $\rho=-0.62 \pm 0.04$ ) (Figure 4c).

\section{CONCLUSIONS}

The LFER determined here suggest that synergistic inhibitors, VA6P and UDP formed an in situ transition state analogue at the active site of OtsA. Thus, the crystal structure of the ternary complex likely reflects the TS of a retaining GT reaction ${ }^{22}$. Several conclusions can be drawn from this TS analogy. Firstly, it suggests that the transition state contains 1) a donor substrate moiety with its leaving group bond largely broken and 2) an acceptor substrate moiety hydrogen bonding between the leaving group oxygen on UDP and the incoming nucleophile (nitrogen in the structure but oxygen in the native substrate). It can now be seen that this hydrogen bonding is an important 
feature of the TS, leading the nucleophile to the same face as the leaving group, as also suggested by a previous computational study ${ }^{18}$. Secondly, we can speculate that the relative distance of the nucleophile might be closer to the anomeric center than that of the leaving group, since the nucleophile-to-carbon bond length is $1.5 \AA$ whilst the interacting UDP is observed $3.5 \AA$ away from the anomeric center in the structure. Clearly, a C-N covalent bond cannot truly mimic the partial bond nature of a TS and indeed an important caveat is that any covalently bonded TS analogues have to be considered imperfect. Subsequently, the distance between UDP and the anomeric carbon determined from this structure might also be an over- or under-estimation. These distances should therefore be taken as a trend of the relative positioning of the leaving group and the nucleophile in the active site. Thirdly, this observed asynchronicity suggested that the oxocarbenium ion-like character is likely featured in the TS. VA6P has a pre-arranged flattened anomeric center around C2-C1-O5-C5 of pyranose ring of the donor mimic. If the reaction proceeded from the ground state to an oxocarbenium ion-like transition state, the geometry of the anomeric center changed from a tetrahedral to a flattened arrangement. This flattened geometry likely contributed to the successful synergistic inhibition of VA6P and UDP as a TS analog. An oxocarbenium ion-like TS in the retaining GT reactions is already implicated in earlier studies that attributed the inhibition of retaining GTs by UDP-2"-deoxy-2"-fluoro-D-glucose or corresponding galactose derivative to the destabilizing effect of an electronegative fluorine adjacent to reaction center on the positively charged $\mathrm{TS}^{10,45}$. Besides geometry, however, the inhibitor structure formed by VA6P and UDP shows less than ideal charge distribution around the putative reaction center since VA6P does not have positive charge on $\mathrm{C} 1$ (numbered as shown in Figure 1 according to VA6P and product trehalose- $6^{\prime}-$ phosphate; $\mathrm{C} 1$ would correspond to $\mathrm{C} 1^{\prime \prime}$ in nucleotide diphosphate donor nomenclature) 
or $\mathrm{C} 7$ (corresponding to endocyclic oxygen $\mathrm{O}^{\prime \prime}$ of the donor pyranose ring) that would develop in a TS. Computational studies on oxocarbenium ion-like transition states of nucleoside glycosylases indicate that the anomeric carbon assumes the most positive charge ${ }^{28}$ Logically and elegantly designed inhibitors of GHs and nucleoside glycosylases mimicking this oxocarbenium ion-like TS incorporate positive charge in these and nearby positions via nitrogen atoms ${ }^{28,46}$. Strong TS analogy of the synergistic inhibitors, despite the lack of proper charge distribution, implied that the majority of the stabilization of positive charge at the anomeric carbon of TS may not be provided by amino acid residues of the enzyme but by the incoming acceptor nucleophile. Indeed in the case of retaining GHs, it is suggested that the stabilization of a positively charged TS is largely provided by the enzymatic nucleophile, a carboxylate group of aspartate or glutamate $^{1,47}$. The LFER determined here are therefore consistent with the lack of an enzymatic nucleophile and indicated that the incoming acceptor nucleophile should play such a role ${ }^{1}$. Therefore, it was necessary to further probe the oxocarbenium ion character of the TS and the role of the nucleophile.

All KIEs pointed to an oxocarbenium ion-like TS with a flattened pyranose ring, and a highly dissociative TS (Figure 3 ). $\left[1{ }^{\prime \prime}-{ }^{14} \mathrm{C}\right] \mathrm{KIE}$ and leaving group $\left[1^{\prime \prime}{ }_{-}^{18} \mathrm{O}\right] \mathrm{KIE}$ suggested that the $\mathrm{C}-\mathrm{O}$ bond to the leaving group was almost broken and that there was asymmetric hydrogen bonding or partial protonation of the leaving group oxygen (or possibly delocalization/non-bridging oxygen protonation $^{42}$ ) at an early stage. Such hydrogen-bonding/protonation of the leaving group was again consistent with the observation of hydrogen bonding in the crystal structure of the ternary complex.

Brønsted relationships in our case could be used to expose the trends of the reaction. Here, more basic hydroxylate groups for the corresponding conjugate nucleophile hydroxyl were found to accelerate the reaction. This was attributed to 
deprotonation of the corresponding conjugate hydroxyl group nucleophile being partial at the TS, which suggested that either deprotonation is at an early stage or the general base in question was not strong. ${ }^{48}$ This was also consistent with the $\left[1^{\prime \prime}{ }_{-}{ }^{18} \mathrm{O}\right] \mathrm{KIE}$ observations described above. Together these indicated a partial protonation of the leaving group oxygen where the leaving group is also the general base. The $\beta_{\text {nuc }}$ of 0.55 indicated simultaneous moderate nucleophilic participation but, at this stage using calculated $\mathrm{pK}_{\mathrm{a}}$ values, it should not be overinterpreted and to assign a particular value to, for example, bond extension would be premature. Taft-like analysis showed the same trends as Brønsted analysis. Generally, when $0>\rho>-1$, it is assumed that the reaction is mildly sensitive to the polar effect of the nucleophile. Our Taft analysis, therefore, also suggested nucleophilic participation in the transition state. It cannot be ruled out that the steric bulk of fluorine substitution might also play a role in modulating rates. If this is the case, it can be speculated that $\rho$ value of -0.62 determined here is a slight overestimation; in that case a lower dependence of the reaction on electronic effects would be expected. This would point towards a more dissociative TS, but one that would still involve participation of the nucleophile. Similar to prior studies with internal donor nucleophiles, ${ }^{49,50}$ our results suggested that for retaining GTs the nucleophile stabilizes positive charge on the anomeric carbon at the highly dissociative transition state by virtue of the lone pair electron of oxygen, not yet making a substantial bond. In this case, the inductive effects observed by us should be read as basicity not as nucleophilicity.

These data, combined with the ${ }^{18} \mathrm{O}$ KIE, supported the existence of hydrogen bonding between the leaving group and the acceptor nucleophile. The role of nucleophile may be to provide the nucleophilic 'push' and the electrostatic stabilization of the cationic anomeric carbon. Also, these LFERs along with the large $\left[1^{\prime \prime}-{ }^{18} \mathrm{O}\right] \mathrm{KIE}$, 
which should arise from contributions up to and including the first irreversible bondbreaking step, strongly suggested that both the leaving group of the donor and the acceptor nucleophile appear in that transition state. This is not consistent with a double displacement mechanism.

Individual experimental sets (e.g. our LFER data that implicate nucleophile participation) may be interpreted as also being consistent with other mechanisms (for example, KIEs alone do not report on reaction stereochemistry); however, together the complete data seemed to provide a less ambiguous set of conclusions. Within the overall context of all of the results of this report, and structural data (Figure 1c), we propose that the reaction catalyzed by a retaining glycosyltransferase, OtsA proceeds via 'same face' nucleophilic substitution (or $\mathrm{S}_{\mathrm{N}} \mathrm{i}$ ), involving a sufficiently "open" transition state that can allow the approach of the nucleophile, Glc6P, guided by hydrogen bonding from the same face as the leaving group (Figure 3), as first proposed $^{17}$ in non-enzymatic reactions. However, whether this proceeds via a metastable intermediate in the reaction coordinate (stepwise $\mathrm{S}_{\mathrm{N}} \mathrm{i} \mathrm{D}_{\mathrm{N}} * \mathrm{~A}_{\mathrm{Nss}}$ ) or the mechanism can be described as concerted (an extremely dissociative $A_{N} D_{N}$ ) remains unclear. This study now provides quantitative data for this unusual mechanism. There are few potent inhibitors of GTs, despite the breadth of this class of enzymes. Their future design (and any associated therapeutic strategies) might be usefully guided by our findings.

\section{METHODS}

OtsA radioactivity stopped assay. Appropriate concentration of UDP-Glc and Glc6P was prepared in $100 \mu \mathrm{L}$ of $100 \mathrm{mM}$ Hepes buffer $(\mathrm{pH} 7.25,100 \mathrm{mM} \mathrm{KCl}, 10 \mathrm{mM}$ $\mathrm{MgCl}_{2}, 0.1 \%$ BSA). Radioactive UDP-[6- $\left.{ }^{3} \mathrm{H}\right]$-Glc (usually 50,000 - 150,000 dpm) was 
added and the reaction mixture was equilibrated at $25^{\circ} \mathrm{C} .5 \mu \mathrm{L}$ of OtsA was added using a syringe to initiate the reaction. After incubation at $25^{\circ} \mathrm{C}$ for $3-10$ minutes, the reaction was stopped by heating briefly ( 30 seconds) at $80{ }^{\circ} \mathrm{C} .400 \mu \mathrm{L}$ of OtsB ( 3 units) in $0.05 \mathrm{mM}$ Tris buffer was added to the mixture. OtsB cleaves trehalose-6-phosphate to trehalose, to produce the only neutral radioactive product in the mixture. The mixture was further incubated for 1 hour at $37^{\circ} \mathrm{C}$ and $\mathrm{pH}$ was adjusted to 8.0. The mixture was then loaded on to pre-conditioned strong anion exchange solid phase extraction Strata ${ }^{\circledR}$ (Phenomenex) cartridge. Flow-through fractions (void volume) and an elution of 100 $\mu \mathrm{L}$ of water were collected in a scintillation counter vial and $5 \mathrm{~mL}$ of liquid scintillation cocktail was added to each vial. Collected solutions were counted by an LS6500 (Beckman-Coulter)liquid scintillation counter. Background samples prepared without OtsA gave no detectable counts.

Kinetic isotope effects. Non-radioactive heavy isotopes such as $\left[1^{\prime \prime}-{ }^{2} \mathrm{H}\right],\left[2^{\prime \prime}-{ }^{2} \mathrm{H}\right]$ and $\left[1^{\prime \prime}-{ }^{18} \mathrm{O}\right]$ were doubly labeled along with $\left[5-{ }^{3} \mathrm{H}\right]$. Consequently, all heavy isotope substrates were ${ }^{3} \mathrm{H}$ labeled except for one occasion. In the case of $\left[1^{\prime \prime}{ }_{-}{ }^{14} \mathrm{C}\right] \mathrm{KIE}, \mathrm{KIE}$ was measured with UDP- $\left[1^{\prime \prime}{ }_{-}{ }^{14} \mathrm{C}\right]-\mathrm{Glc}$ as the heavy isotope substrate and UDP-[6" $\left.{ }^{3} \mathrm{H}\right]-$ Glc as the light isotope substrate, and the observed value was corrected for the remote effect from $\left[6^{\prime \prime}{ }_{-}^{3} \mathrm{H}\right]\left(\left[1^{\prime \prime}-{ }^{14} \mathrm{C}\right] \mathrm{KIE}=\left[1^{\prime \prime}{ }_{-}{ }^{14} \mathrm{C}\right] \mathrm{KIE}\right.$ (observed) $\left.\times\left[6^{\prime \prime}-{ }^{3} \mathrm{H}\right] \mathrm{KIE}\right)$. A master reaction mix was prepared using 2 mM UDP-Glc, 8 mM 2-deoxy-D-glucose6-phosphate, $50 \mathrm{mM} \mathrm{KCl}, 10 \mathrm{mM} \mathrm{MgCl} 2,3$ units of OtsB and $0.1 \% \mathrm{BSA}$ in $50 \mathrm{mM}$ Hepes buffer, $\mathrm{pH} 7.2$ containing isotopic pairs of radiolabeled UDP-Glc substrates whose ratio of ${ }^{3} \mathrm{H} /{ }^{14} \mathrm{C}$ was ca $1: 1$ with an activity of ca $100,000 \mathrm{dpm}$ each. Enzymatic reaction was initiated by taking aliquots from the master mix and adding OtsA to each aliquot. ${ }^{3} \mathrm{H} /{ }^{14} \mathrm{C}$ ratio $\left(\right.$ or ${ }^{14} \mathrm{C} /{ }^{3} \mathrm{H}$ ) from the master mix was measured and taken as $\mathrm{R}_{0}$. The enzymatic reaction was incubated at $25{ }^{\circ} \mathrm{C}$ and allowed to proceed until $40-50 \%$ 
completion. The reaction was stopped by freezing the mixture with liquid nitrogen. Small aliquots $(20 \mu \mathrm{L})$ were taken for HPLC analysis of the reaction fraction (f). The unreacted product was purified by HPLC on an UltiMate 3000 System (Dionex) using anion exchange column Spherisorb ${ }^{\circledR} \mathrm{SAX}(10 \times 250 \mathrm{~mm}$, Waters $)$. The column was eluted with a gradient of $15-300 \mathrm{mM}$ ammonium formate (30 minutes), $\mathrm{pH} 3.5$ and chromatography was monitored by UV detector at $262 \mathrm{~nm}$. The unreacted substrate was eluted at the retention time of 18.5 minutes, which was pooled and lyophilized. The residue was dissolved in $200 \mu \mathrm{L}$ water and subsequently mixed with $5 \mathrm{~mL}$ scintillation cocktail. Liquid scintillation counting was performed on a Liquid Scintillation Counter LS6500 (Beckmann-Coulter), preset to read ${ }^{3} \mathrm{H} /{ }^{14} \mathrm{C}$ ratio with a precision of $0.2 \%$. Each counting set was performed for 10 minutes. Resulting ratios ${ }^{3} \mathrm{H} /{ }^{14} \mathrm{C}$ (or ${ }^{14} \mathrm{C} /{ }^{3} \mathrm{H}$ in the case of $\left[1^{\prime \prime}-{ }^{14} \mathrm{C}\right] \mathrm{KIE}$ ) were taken as $\mathrm{R}_{\mathrm{t}}$. The fraction for reaction (f) was obtained by HPLC analysis comparing the ratio of the peak areas of the substrate UDP-Glc and product UDP with a standard curve measured with a UV detector at $262 \mathrm{~nm}$ (column: Waters Spherisorb ${ }^{\circledR}$ SAX $4.6 \times 250 \mathrm{~mm}$ using a gradient of $25-250 \mathrm{mM} \mathrm{NaH} \mathrm{PO}_{4}$ for 35 minutes). KIE was calculated using the following equation.

$$
K I E=\frac{\log (1-f)}{\log \left[(1-f)^{\left.R_{t} / R_{0}\right]}\right.} \quad \text { Eqn } 1
$$

$\mathrm{f}=$ the fraction reaction $(0-1)$ at time when the reaction was stopped.

$\mathrm{R}_{\mathrm{t}}={ }^{3} \mathrm{H} /{ }^{14} \mathrm{C}$ (or ${ }^{14} \mathrm{C} /{ }^{3} \mathrm{H}$ ) of unreacted substrate when the reaction was stopped (partial reaction) $\mathrm{R}_{0}={ }^{3} \mathrm{H} /{ }^{14} \mathrm{C}$ (or ${ }^{14} \mathrm{C} /{ }^{3} \mathrm{H}$ ) of total substrate (ratio at time zero).

$\left[1^{\prime \prime}-{ }^{18} \mathrm{O}\right]$ KIE was further corrected by percent enrichment of ${ }^{18} \mathrm{O}$ incorporation by using the following equation ${ }^{30}$. The ${ }^{18} \mathrm{O}$ fraction $(0.824)$ in $\alpha$-D-glucopyranosyl-[1- $\left.{ }^{18} \mathrm{O}\right]-$ 
phosphate was taken as $\mathrm{f}_{\text {iso }}$ value since this compound was completely converted to the final product $\left[{ }^{5} \mathrm{H}\right]-\mathrm{UDP}-\left[1^{\prime \prime}-{ }_{-}^{18} \mathrm{O}\right]-\mathrm{Glc}$ in the enzymatic synthesis.

$\mathrm{KIE}_{\text {final }}=\left(\mathrm{KIE}_{\text {measured }}-1+\mathrm{f}_{\text {iso }}\right) / \mathrm{f}_{\text {iso }} \quad$ Eqn2

$\mathrm{KIE}_{\text {final }}=$ final ${ }^{18} \mathrm{O} \mathrm{KIE}$

$\mathrm{KIE}_{\text {measured }}=\mathrm{KIE}$ determined by the equation Eqn 1

$\mathrm{f}_{\mathrm{iso}}=$ the percent isotopic fraction of ${ }^{18} \mathrm{O}$

Data Fitting and Statistics. All data (from typically three individual experiments) were analysed through linear regression using either the Michaelis-Menten equation (for kinetics), Dixon plot (for inhibition) and those for LFER or through non-linear regression using bisubstrate kinetic equations in GraFit 7.0 (2011, Erithacus Software, East Grinstead, UK) and this regression generated standard errors of means (s.e.m.). 


\section{Acknowledgements}

We thank the Bill and Melinda Gates Foundation, the Samsung Fellowship and the BBSRC for financial support. Dr Ran Zhang and Prof. Stephen G. Withers are acknowledged for providing 2-deoxy-2,2-difluoro-glucose. BGD and GJD are both

Royal Society Wolfson Research Merit Award recipients and BGD is supported by an EPSRC LSI Platform grant. 


\section{Author Contributions}

SSL, BGD designed the experiments. AI provided mutant ots $A$ plasmids. SSL, SYH, JCE expressed the mutants. SSL, JCE performed the kinetic measurements. SSL

performed all other experiments. SSL, BGD, GJD analyzed the experiments. BGD, SSL, GJD wrote the manuscript.

\section{Competing Financial Interests Statement}

The authors declare no competing financial interests. 


\section{References}

1. Lairson, L.L., Henrissat, B., Davies, G.J. \& Withers, S.G. Glycosyltransferases: Structures, Functions and Mechanisms. Annu. Rev. Biochem. 77, 521-555 (2008).

2. Kim, S.C., Singh, A.N. \& Raushel, F.M. Analysis of the Galactosyltransferase Reaction by Positional Isotope Exchange and Secondary Deuterium Isotope Effects. Arch. Biochem. Biophys. 267, 54-59 (1988).

3. Bruner, M. \& Horenstein, B.A. Isotope Trapping and Kinetic Isotope Effect Studies of Rat Liver $\alpha$-(2-6)-Sialyltransferase. Biochemistry 37, 289-297 (1998).

4. Vocadlo, D.J., Davies, G.J., Laine, R. \& Withers, S.G. Catalysis by hen eggwhite lysozyme proceeds via a covalent intermediate. Nature 412, 835-838 (2001).

5. Lairson, L.L. et al. Intermediate trapping on a mutant retaining alphagalactosyltransferase identifies an unexpected aspartate residue. J. Biol. Chem. 279, 28339-28344 (2004).

6. Soya, N., Fang, Y., Palcic, M.M. \& Klassen, J.S. Trapping and Characterization of Covalent Intermediates of Mutant Retaining Glycosyltransferases.

Glycobiology 21, 547-552 (2011).

7. Monegal, A. \& Planas, A. Chemical Rescue of a3-Galactosyltransferase. Implications in the Mechanism of Retaining Glycosyltransferase. J. Am. Chem. Soc. 128, 16030-16031 (2006).

8. Ly, H.D., Lougheed, B., Wakarchuk, W.W. \& Withers, S.G. Mechanistic Studies of a Retaining a-Galactosyltransferase from Neisseria meningitidis. Biochemistry 41, 5075-5085 (2002).

9. Gibson, R.P., Turkenburg, J.P., Charnock, S.J., Lloyd, R. \& Davies, G.J. Insights into Trehalose Synthesis Provided by the Structure of the Retaining Glucosyltransferase OtsA. Chem. Biol. 9, 1337-1346 (2002).

10. Persson, K. et al. Crystal structure of the retaining galactosyltransferase LgtC from Neisseria meningitidis in complex with donor and acceptor analogs. Nature Struct. Biol. 8, 166-175 (2001).

11. Gastinel, L.N. et al. Bovine $\alpha$-1,3-galactosyltransferase catalytic domain structure and its relationship with $\mathrm{ABO}$ histo-blood group and glycosphingolipid glycosyltransferase. EMBO J. 20, 638-649 (2001).

12. Patenaude, S.I. et al. The structural basis for specificity in human $\mathrm{ABO}(\mathrm{H})$ blood group biosynthesis. Nature Struct. Biol. 9, 685-690 (2002).

13. Martinez-Fleites, C. et al. Insights into the Synthesis of Lipopolysaccharide and Antibiotics through the Structures of Two Retaining Glycosyltransferases from Family GT4. Chem. Biol. 13, 1143-1152 (2006).

14. Jamaluddin, H., Tumbale, P., Withers, S.G., Acharya, K.R. \& Brew, K. Conformational Changes Induced by Binding UDP-2F-galactose to alpha-1,3Galactosyltransferase - Implications for Catalysis. J. Mol. Biol. 369, 1270-1281 (2007).

15. Vetting, M.W., Frantom, P.A. \& Blanchard, J.S. Structural and Enzymatic Analysis of MshA from Corynebacterium glutamicum. J. Biol. Chem. 283, 15834-15844 (2008).

16. Cowdrey, W.A., Hughes, E.D., Ingold, C.K., Masterman, S. \& Scott, A.D. 257. Reaction kinetics and the Walden inversion. Part VI. Relation of steric 
orientation to mechanism in substitutions involving halogen atoms and simple or substituted hydroxyl groups. J. Chem. Soc., 1252-1271 (1937).

17. Sinnott, M.L. \& Jencks, W.P. Solvolysis of D-Glucopyranosyl Derivatives in Mixtures of Ethanol and 2,2,2-Trifluoroethanol. J. Am. Chem. Soc. 102, 20262032 (1980).

18. Tvaroska, I. Molecular modeling of retaining glycosyltransferases. ACS Symposium Series 930, 285-301 (2006).

19. Nidetzky, B. \& Eis, C. a-Retaining glucosyl transfer catalysed by trehalose phosphorylase from Schizophyllum commune: mechanistic evidence obtained from steady-state kinetic studies with substrate analogues and inhibitors. Biochem. J. 360, 727-736 (2001).

20. Goedl, C., Griessler, R., Schwarz, A. \& Nidetzky, B. Structure-function relationships for Schizophyllum commune trehalose phosphorylase and their implications for the catalytic mechanism of family GT-4 glycosyltransferase. Biochem. J. 397, 491-500 (2006).

21. Goedl, C. \& Nidetzky, B. Sucrose Phosphorylase Harboring a Redisigned, Glycosyltransferase-Like Active Site Exhibits Retaining Glucosyl Transfer in the Absence of a Covalent Intermediate. ChemBioChem 10, 2333-2337 (2009).

22. Errey, J.C. et al. Mechanistic Insight into Enzymatic Glycosyl Transfer with Retention of Configuration through Analysis of Glycomimetic Inhibitors. Angew. Chem Int. Ed. 49, 1234-1237 (2010).

23. Coutinho, P.M., Deleury, E., Davies, G.J. \& Henrissat, B. An Evolving Hierarchical Family Classification for Glycosyltransferases. J. Mol. Biol. 328(2003).

24. Greig, I.R. The analysis of enzymic free energy relationships using kinetic and computational models. Chem. Soc. Rev. 39, 2272-2301 (2010).

25. Mader, M.M. \& Bartlet, P.A. Binding Energy and Catalysis: The Implications for Transition-State Analogs and Catalytic Antibodies. Chem. Rev. 97, 12811301 (1997).

26. Gosselin, S., Alhussaini, M., Streiff, M.B., Takabayashi, K. \& Palcic, M.M. A continuous spectrophotometric assay for glycosyltransferases. Anal. Biochem. 220, 92-97 (1994).

27. Baxter, N.J. et al. A Trojan horse transition state analogue generated by $\mathrm{MgF}_{3}^{-}$ formation in an enzyme active site. Proc. Natl. Acad. Sci., USA 103, 1473214737 (2006).

28. Berti, P.J. \& McCann, J.A.B. Toward a Detailed Understanding of Base Excision Repair Enzymes: Transition State and Mechanistic Analyses of NGlycoside Hydrolysis and N-Glycosyl Transfer. Chemical Reviews 106, 506-555 (2006).

29. Northrop, D.B. Minimal Kinetic Mechanism and General Equation for Deuterium Isotope Effects on Enzymic Reactions: Uncertaintly in Detecting a Rate-Limiting Step. Biochemistry 20, 4056-4061 (1981).

30. Werner, R.M. \& Stivers, J.T. Kinetic Isotope Effects Studies of the Reaction Catalyzed by Uracil DNA Glycosylase: Evidence for an Oxocarbenium IonUracil Anion Intermediate. Biochemistry 39, 14054-14064 (2000).

31. Luo, M. \& Schramm, V.L. Transition State Structure of E. coli tRNA-Specific Adenosine Deaminase. J. Am. Chem. Soc. 130, 2639-2655 (2008).

32. Cleland, W.W. The use of isotope effects to determine enzyme mechanisms. Arch. Biochem. Biophys. 433, 2-12 (2005). 
33. Lee, J.K., Bain, D.A. \& Berti, P.J. Probing the Transition States of Four Glucoside Hydrolyses with ${ }^{13} \mathrm{C}$ Kinetic Isotope Effects Measured at Natural Abundance by NMR Spectroscopy. J. Am. Chem. Soc. 126, 3769-3776 (2004).

34. Glad, S.S. \& Jensen, F. Transition State Looseness and $\alpha$-Secondary Kinetic Isotope Effects. J. Am. Chem. Soc. 119, 227-232 (1997).

35. Sunko, D.E., Szele, I. \& Hehre, W.J. Hyperconjugation and the Angular Dependence of $\beta$-Deuterium Isotope Effects. J. Am. Chem. Soc. 99, 5000-5005 (1977).

36. Guthrie, R.D. \& Jencks, W.P. IUPAC Recommendations for the Representation of Reaction Mechanisms. Acc. Chem. Res. 22, 343-349 (1989).

37. Huang, X., Tanaka, K.S.E. \& Bennet, A.J. Glucosidase-Catalyzed Hydrolysis of $\alpha$-D-Glucopyranosyl Pyridinium Salts: Kinetic Evidence for Nucleophilic Involvement at the Glucosidation Transition State. J. Am. Chem. Soc. 119, 11147-11154 (1997).

38. Chan, J., Lewis, A.R., Gilbert, M., Karwaski, M.-F. \& Bennet, A.J. A direct NMR method for the measurement of competitive kinetic isotope effects. Nature Chem. Biol. 6, 405-407 (2010).

39. Rosenberg, S. \& Kirsch, J.F. Oxygen-18 Leaving Group Kinetic Isotope Effects on the Hydrolysis of Nitrophenyl Glycosides. 2. Lysozyme and betaGlucosidase: Acid and Alkaline Hydrolysis. Biochemistry 20, 3196-3204 (1981).

40. Bennet, A.J. \& Sinnott, M.L. Complete kinetic isotope effect description of transition states for acid-catalyzed hydrolyses of methyl alpha- and betaglucopyranosides. J. Am. Chem. Soc. 108, 7287-7294 (1986).

41. Indurugalla, D. \& Bennet, A.J. A Kinetic Isotope Effect Study on the Hydrolysis Reactions of Methyl Xylopyranosides and Methyl 5-Thioxylopyranosides: Oxygen versus Sulfur Stabilization of Carbenium Ions. J. Am. Chem. Soc. 123, 10889-10898 (2001).

42. Du, X., Black, G.E., Lecchi, P., Abramson, F.P. \& Sprang, S.R. Kinetic isotope effects in Ras-catalyzed GTP hydrolysis: Evidence for a loose transition state. Proc. Natl. Acad. Sci., USA 101, 8858-8863 (2004).

43. Chenault, H.K., Mandes, R.F. \& Hornberger, K.R. Synthetic Utility of Yeast Hexokinase. Substrate Specificity, Cofactor Regeneration, and Product Isolation. J. Org. Chem. 62, 331-336 (1997).

44. Hansch, C. \& Leo, A. Substituent constants for correlation analysis in chemistry and biology, (John Wiley \& Sons, Inc., New York, 1979).

45. Gibson, R.P., Tarling, C.A., Roberts, S., Withers, S.G. \& Davies, G.J. The Donor Subsite of Trehalose-6-phosphate Synthase. J. Biol. Chem. 279, 1950 1955 (2004).

46. Gloster, T.M. \& Davies, G.J. Glycosidase inhibition: assessing mimicry of the transition state. Org. Biomol. Chem. 8, 305-320 (2010).

47. Sinnott, M.L. Catalytic Mechanism of Enzymic Glycosyl Transfer. Chemical Reviews 90, 1171-1202 (1990).

48. Ye, J.-D., Li, N.-S., Dai, Q. \& Piccirilli, J.A. The Mechanism of RNA Strand Scission: An Experiemtnal Measure of the Bronsted Coefficient, $\beta_{\text {nuc. Angew. }}$. Chem Int. Ed. 46, 3714-3717 (2007).

49. Jones, C.S. \& Kosman, D.J. Purification, Properties, Kinetics, and Mechanism of $\beta$-N-Acetylglucosaminidase from Aspergillus niger. J. Biol. Chem. 255, 11861-11869 (1980). 
50. Macauley, M.S., Whitworth, G.E., Debowski, A.W., Chin, D. \& Vocadlo, D.J. O-GlcNAcase Uses Substrate-assisted Catalysis. J. Biol. Chem. 280, 2531325322 (2005). 
Figure 1. The reaction catalyzed by trehalose-6-phosphate synthase (OtsA). The numbering shown here is for both VA6P and the product trehalose- 6 '-phosphate (and when discussing these compounds in the main text) to allow consistent numbering through the reaction pathway. Discussion of the nucleotide donor in the main text uses conventional numbering (as shown in Table 1); for example, $\mathrm{C} 1$ in VA6P corresponds to $\mathrm{Cl}^{\prime \prime}$ in the nucleotide diphosphate donor. (a) The reaction forms a glycosidic bond between the $\mathrm{OH}^{\prime}$-hydroxyl of acceptor substrate glucose-6-phosphate (blue) and the glucosyl moiety (red) from donor substrate UDP-glucose (UDPGlc). (b) The proposed front-face transition state for this reaction resembles (c) The complex ternary structure (right) determined ${ }^{22}$ for the synergistic binding to OtsA of UDP and validoxylamine A 6'-O-phosphate (VA6P) with corresponding schematic view (left). Each mutation generated for LFER is shown in parentheses in the schematic view. (d) The structure of VA6P. Note, the secondary amine in VA6P might be expected to be protonated at physiological $\mathrm{pH}$ but this is not clearly observed (either directly or indirectly) in determined structures. 
Figure 2. Linear Free Energy Relationships of OtsA Variants. (a) Plot of log $\left(\mathrm{K}_{\mathrm{m}} / \mathrm{k}_{\mathrm{cat}}\right)$ of OtsA wildtype and mutants versus $\log \mathrm{K}_{\mathrm{i}}$ measured with VA6P in the presence of UDP at concentration of twice $K_{i}$ of UDP. The slope $=0.98\left(r^{2}=0.94\right)$ strongly suggests effective mimicry of the enzyme TS by the synergistic inhibition of VA6P and UDP. (b) Plot of $\log K_{\mathrm{i}}$ versus $\log \left(K_{\mathrm{m}} / k_{\mathrm{cat}}\right)$ of OtsA wildtype and mutants measured with VA6P alone in the absence of UDP. $K_{\mathrm{i}}$ values for two mutants (D130A and D130N) could not be measured since $K_{\mathrm{i}}$ values were very high, out of range of the concentration of VA6P used (up to $160 \mathrm{mM}$ ). A poor relationship $\left(\right.$ slope $=0.63, \mathrm{r}^{2}=$ 0.79) suggests that VA6P is only a poor (or not at all a) TS mimic in the absence of UDP. (c) Plot of $\log K_{\mathrm{i}}$ versus $\log \left(K_{\mathrm{m}} / k_{\mathrm{cat}}\right)$ of OtsA wildtype and mutants measured with UDP alone in the absence of VA6P. As for (b) a lack of correlation suggests that VA6P is is not an effective TS mimic in the absence of VA6P. Three independent measurements were performed for each data point. 


\section{Figure 3. A schematic diagram depicting the profile (energy vs coordinate) of OtsA}

catalyzed reaction deduced from experiments. Binding of substrates occurs sequentially (ordered bi-bi), the donor first, followed by the acceptor. As the reaction reaches the transition state, the following series of events occur. The leaving group departure is almost completed as the nucleophile starts to form a significant bond. This confers the TS a substantial oxocarbenium ion character. Consequently, the pyranose ring of the donor is flattened through $\mathrm{C}^{\prime \prime}-\mathrm{O} 5^{\prime \prime}-\mathrm{C}^{\prime \prime}-\mathrm{C} 2^{\prime \prime}$. The oxygen atom of the leaving group accepts a hydrogen bond from the nucleophile, and this ensures the presence of the nucleophile along the reaction coordinate. Combined with the Brønsted and Taft-like analysis, the participation of nucleophile seems to be significant. The mechanism can be either concerted or step-wise. The TS is highly dissociative in either and the energy difference between the two scenarios is expected to be small. If there is a discrete intermediate, the nucleophile contributes to the stabilization of the intermediate. 
Figure 4. LFER of acceptor kinetics determined using the varied modified acceptors. (a) Panel of modified deoxy- and deoxy-fluoro-acceptor substrates (2-9) of OtsA used to probe the linear free energy relationships of acceptor substrate kinetics. (b) Brønsted relationship of nucleophile basicity and rates. Plot of calculated (see Supplementary Methods for further details) $\mathrm{pK}_{\mathrm{a}}$ of nucleophile versus $\log \left(\mathrm{k}_{\mathrm{cat}} / \mathrm{K}_{\mathrm{m}}\right)$. Slope $\left(\beta_{\text {nuc }}\right)=0.54, r^{2}=0.98$. (c) Taft-like analysis. Plot of polar substituent constants $\left(\sigma^{*}\right)$ versus $\log \left(\mathrm{k}_{\mathrm{cat}} / \mathrm{K}_{\mathrm{m}}\right)$. Slope $(\rho)=0.62, \mathrm{r}^{2}=1.0$. Three independent measurements were performed for each data point. 
Table 1. The position of isotopic labels in the donor substrate, UDP-Glc, designed for multiple kinetic isotope effect measurement and the resulting experimental KIEs that were determined. Three to five independent measurements were performed for each KIE and data represent mean values \pm s.d.

\begin{tabular}{|c|c|c|}
\hline Label & Type of KIE & Experimental KIE $^{\mathrm{a}}$ \\
\hline $\mathbf{1}^{\mathbf{}}-{ }^{\mathbf{3}} \mathbf{H}$ & $\alpha$-secondary & $1.284( \pm 0.001)$ \\
\hline $\mathbf{1}^{\prime \prime}-{ }^{2} \mathbf{H}$ & $\alpha$-secondary & $1.196( \pm 0.003)$ \\
\hline $\mathbf{1}^{\prime \prime}-{ }^{14} \mathbf{C}$ & primary label & $1.023( \pm 0.004)^{\mathrm{b}}$ \\
\hline $\mathbf{2}^{\mathbf{}}-{ }^{2} \mathbf{H}$ & $\beta$-secondary & $1.164( \pm 0.003)$ \\
\hline $\mathbf{1}^{\prime \prime}-{ }^{\mathbf{1}} \mathbf{O}$ & primary; leaving group & $1.024( \pm 0.002)$ \\
\hline $\mathbf{6}^{\prime \prime}-{ }^{3} \mathbf{H}$ & remote label & $1.049( \pm 0.0002)$ \\
\hline $\mathbf{5}^{\mathbf{3}} \mathbf{H}$ & remote label & $1.000( \pm 0.004)$ \\
\hline
\end{tabular}

${ }^{\text {a }}$ All measured with 2-deoxy-glucose-6-phosphate as an acceptor substrate

${ }^{\mathrm{b}}$ Corrected for remote label effect of $\left[6^{\prime \prime}-{ }^{3} \mathrm{H}\right]$ 


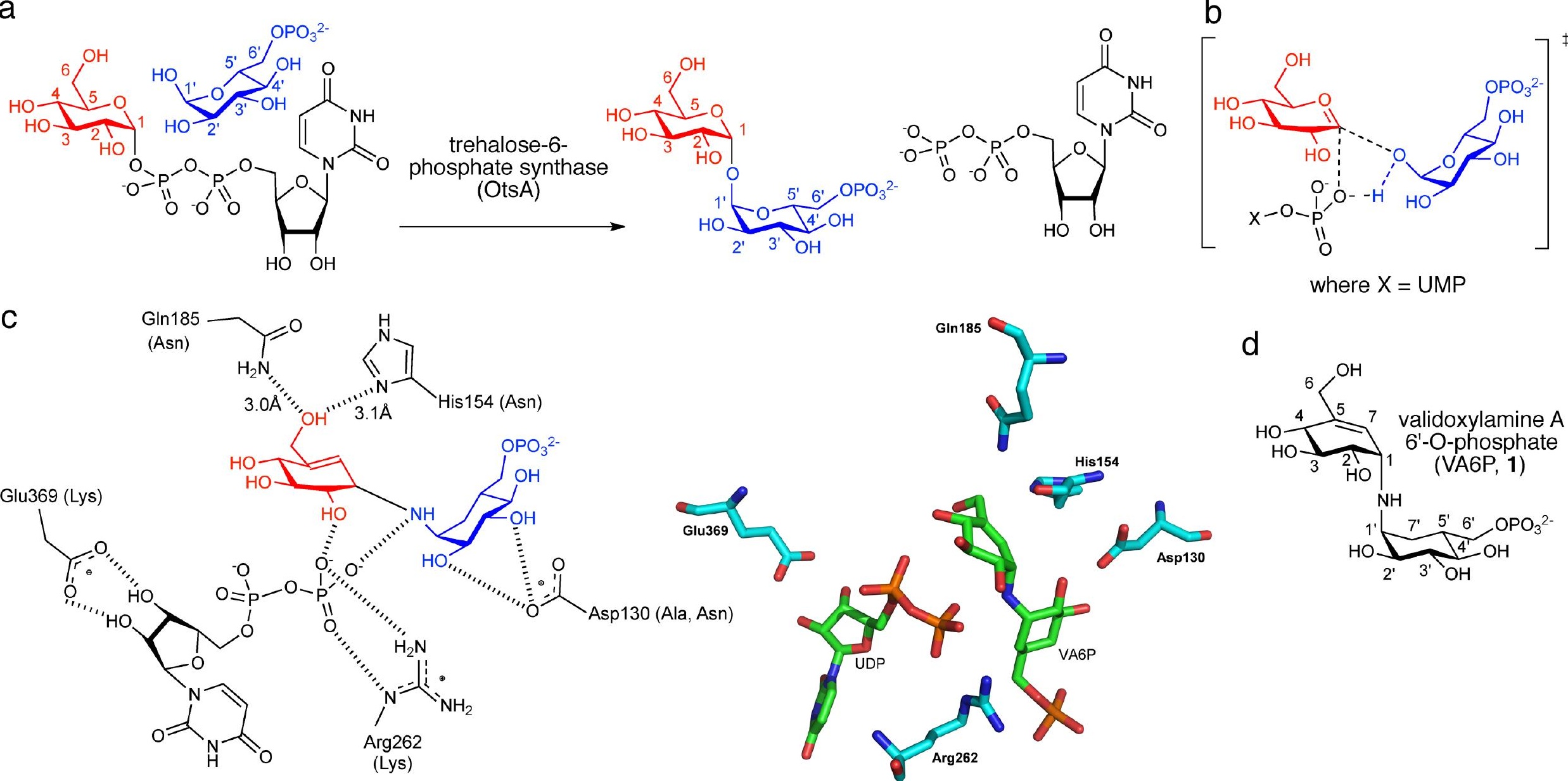



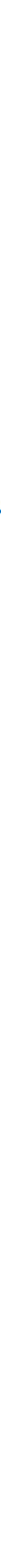

$0 \quad 2$ 

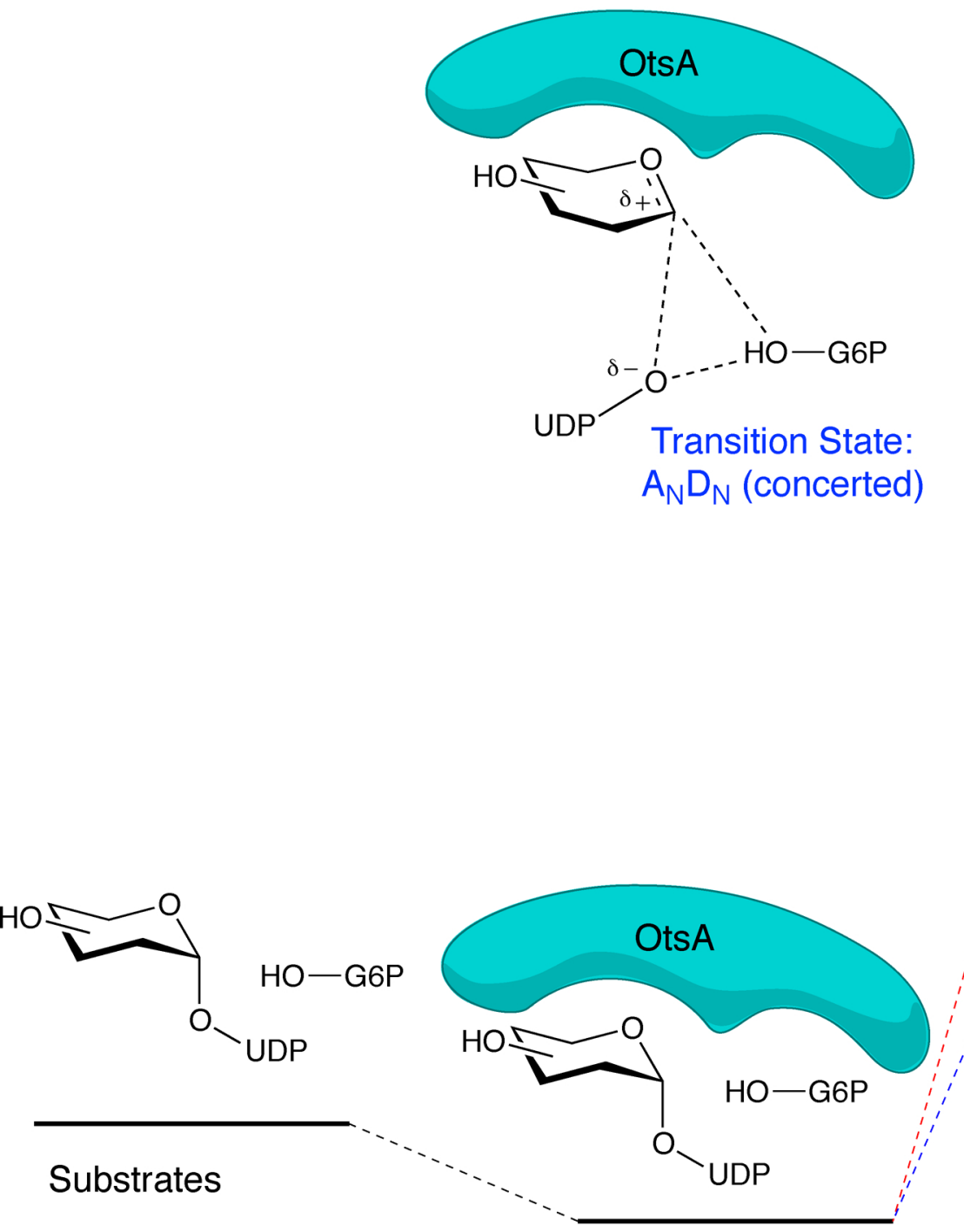

Michaelis complex 


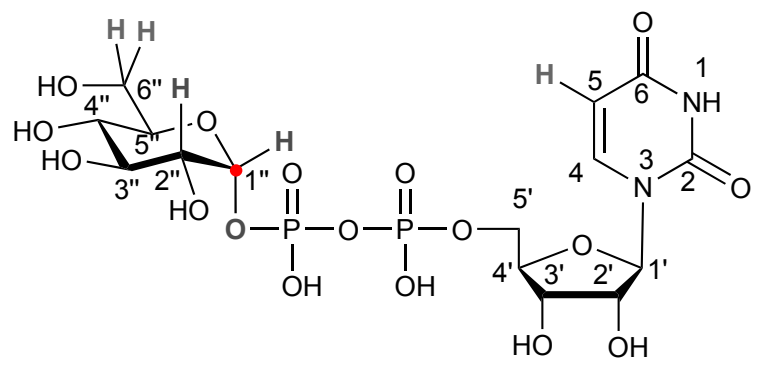

